\title{
Dynamics of Dissipative Viscous Cylindrical Collapse with Full Causal Approach in $f(R)$ Gravity
}

\author{
G. Abbas $(\mathbb{D})$ and H. Nazar \\ Department of Mathematics, The Islamia University of Bahawalpur, Bahawalpur, Pakistan \\ Correspondence should be addressed to G. Abbas; abbasg91@yahoo.com
}

Received 3 September 2018; Revised 28 September 2018; Accepted 2 October 2018; Published 24 October 2018

Guest Editor: Subhajit Saha

Copyright (C) 2018 G. Abbas and H. Nazar. This is an open access article distributed under the Creative Commons Attribution License, which permits unrestricted use, distribution, and reproduction in any medium, provided the original work is properly cited. The publication of this article was funded by SCOAP ${ }^{3}$.

\begin{abstract}
The idea of this article is to examine the effects on dynamics of dissipative gravitational collapse in nonstatic cylindrical symmetric geometry by using Misner-Sharp concept in framework of metric $f(R)$ gravity theory. In this interest, we extended our study to the dissipative dark source case in both forms of heat flow and the free radiation streaming. Moreover, the role of different quantities such as heat flux, bulk, and shear viscosity in the dynamical equation is evaluated in thorough version. The dynamical equation is then coupled with full causal transportation equations in the context of Israel-Stewart formalism. The present scheme explains the physical consequences of the gravitational collapse and that is given in the decreasing form of inertial mass density which depends on thermodynamics viscous/heat coupling factors in background of $f(R)$ theory of gravity. It is very interesting to tell us that the motives of this theory are reproduced for $f(R)=R$ into general theory of relativity that has been done earlier.
\end{abstract}

\section{Introduction}

A multiple work has been done in the significance of relativistic geometry of the collapsing star to the general relativity theory and higher order curvature theories. In this scenario, too many open challenges and curious issues still exist in modern physics, gravitational physics, cosmological physics, relativistic dense objects, group of galaxies, neutron star, and black holes. Currently, our aim is to discuss the gravitational collapse of the relativistic dense object in the presence of dissipative term.

In this arrangement, the formation of the dense star is highly explosive and exhaustible due to the highly inmost pull of gravity and this process is terminated with the external pressure of the interior fuel. However, once the object has wakened due to its thermodynamical fission reaction, there is no any extended thermodynamical burning and there will be an interminable gravitating collapse. The geometry of the object is made up by its explosive mass which is constantly gravitational and it is stimulating by the interior core of the object due to the gravitating interaction between their particles. Oppenheimer and Snyder [1] made an activity stride in the field of gravitating collapse and examined the nitty gritty work in regard to the gravitational collapse issues. In this order after the midway of the 20th century, a brief sensible evaluation was recognized by Misner and Sharp [2] with isotropic matter distribution for the interior of the imploding object as well as in vacuum of the outside object. Vaidya [3] explained the gravitational collapse with the radiating source.

Numerous models still exist to explain the enigmatic role of the dark energy in a system. In this inference, we use the higher order modified gravity theories such as $f(R)$, $f(G)$, and $f(R, T)$ gravity theories; it can provide the wellestablished framework in the significance of rushing evolution of the universe. Capozziello [4] studied the notable issues for the accelerated growth of the universe and quintessence in the Friedmann Robertson Walker (FRW) model with higher curvature gravity theories. Sharif and Yousaf [5] explored the stability of the anisotropic fluid distribution in nonstatic spherically symmetric geometry with $f(R)$ metric formalism. Mak and Harko [6] investigated the notional indications through many astrophysical estimations and found the significant natural dissimilarities in the pressure. Herrera and Santos [7] determined the impacts on imperfect fluid formation with astral spherical object for the behavior of restful rotation. 
Webber [8] examined the solutions of pressure anisotropy in dense models with electromagnetic field. Chakraborty et al. [9] analyzed the gears of tangential pressure and along with radial pressure in stellar structures by quasispherical paradigms in the form of collapse. Garattini [10] evaluated the formation of exposed singularity through mass limitation in metric $f(R)$ theory of gravity. Sharif and his coresearchers [11-18] studied the various consequences of energy density inhomogeneity and Weyl tensor with gravitational collapse in dense objects with General relativity theory as well as in modified theories of gravity. Cognola et al. [19] introduced the results of spherical imploding object for the black hole in $f(R)$ context by the parameter of positive Ricci invariant $R$. Capozziello et al. [20] examined the modified Lané-Emden equation in context of metric $f(R)$ gravity theory and studied the hydrostatic periods of astral models. Copeland et al. [21] analyzed the several concepts for the rushing growth of universe. Amendola et al. [22] discussed the feasible paradigms of metric $f(R)$ gravity in the context of both Einstein and Jordan frames.

Chandrasekhar [23] introduced the impacts on the constancy of dynamics with perfect fluid collapsing geometry. Herrera et al. [24] discussed the results of dynamical disequilibrium with spherically symmetric nonadiabatic collapse and found the heat flow out from the gravitating source in the form of radial heat flux. Abbas et al. [25] presented the results of compact star models in anisotropic fluid distribution with cylindrical symmetric static spacetime geometry. Mak and Harko [26] studied the kind of exact solutions of field equations by considering the spherical symmetric structure and also conferred the energy density along with tangential and radial pressure that are limited and increased in the core of the imperfect fluid object. Rahaman et al. [27] prolonged the Krori-Barua solution with spherical symmetric static spacetime for the investigation of charge imperfect fluid distribution. Herrera and his collaborators [28-33] did work in different directions, such as self-gravitating dens models discussed with local anisotropic matter distribution in the cracking spherical symmetric structure in context of equation of state with perturbed approach. They established the various results in gravitational collapse for the dissipative case by using Misner and Sharp approach; such gravitating source undergoes in form of free radiation streaming and diffusion approximations. They investigated the expansion free conditions for the spherically symmetric anisotropic dissipative collapsing matter. Further, Herrera and his collaborators [3438] have evaluated the Lake algorithm in static spherically symmetric anisotropic configuration for the determination of new formalism of two functions (instead of one) to generate all possible results. They studied the results of structure scalars $Y_{T F}$ for the charged dissipative anisotropic collapsing geometry in presence of cosmological constant. They have also done detailed work on the dynamical disequilibrium with spherically symmetric locally anisotropic fluid which collapses adiabatically under the expansion-free condition. In few investigations they have used the noncasual as well as casual approaches to discuss the dynamics of gravitating source. Nolan [39] examined the effects on gravitational collapse for counter rotating dust cloud with cylindrical paradigms and found the naked singularity. Hayward [40] produced the results of cylindrical geometry for black holes, gravitational waves, and cosmic strings.

In this paper, we analyze the self-gravitational collapse with cylindrical symmetric geometry in context of metric $f(R)$ gravity theory. The main persistence of this study is to examine the viscous dissipative collapse in the form of radial heat flow and free radiation streaming in cylindrical nonstatic spacetime with full causal approach.

The proposal of this work is as follows. In Section 2, we express the cylindrical symmetric isotropic collapsing matter distribution and its related variables in context of $f(R)$ theory of gravity. Section 3 consists of Einstein modified field equations; matching conditions are given for the interior and exterior manifolds $M^{-}$and $M^{+}$and the dynamical equation that was accompanied with physical parameters such as heat flux, bulk, and shear viscosity. Section 4 is devoted to the transportation equations attained in frame of Müller-IsraelStewart formalism [41-43]; then transportation equations are associated with dynamical equation. Finally, the conclusions of the study have been discussed in the last section.

\section{Self-Gravitational Collapsing Geometry and Related Conventions}

In this section, we consider a locally isotropic collapsing fluid with full causal description in context of MisnerSharp approach, formed by cylindrically symmetric nonstatic interior and exterior geometry of the dissipative fluid which undergoes dissipation in the form of heat flow and free streaming radiation. In general relativity the Einstein-Hilbert $(\mathrm{EH})$ action can be expressed as follows:

$$
S_{E H}=\frac{1}{2 \kappa} \int d^{4} x \sqrt{-g} R .
$$

The extended formulation of the Einstein-Hilbert $(\mathrm{EH})$ in $f(R)$ context is

$$
S_{\text {modif }}=\frac{1}{2 \kappa} \int d^{4} x \sqrt{-g}\left(f(R)+L_{(\text {matter })}\right),
$$

where $f(R)$ is arbitrary function of Ricci scalar $R, \kappa$ is coupling constant, and $L_{\text {(matter) }}$ the Lagrangian matter density of the action. The following modified $f(R)$ field equations are obtained by the variation of (2) with respect to $g_{\alpha \beta}$ :

$$
\begin{aligned}
& F(R) R_{\alpha \beta}-\frac{1}{2} f(R) g_{\alpha \beta}-\nabla_{\alpha} \nabla_{\beta} F(R)+g_{\alpha \beta} \nabla^{\alpha} \nabla_{\alpha} F(R) \\
& =\kappa T_{\alpha \beta},
\end{aligned}
$$

where $F(R)=d f(R) / d R, \nabla^{\alpha} \nabla_{\alpha}$ is the D' Alembert operator, $\nabla_{\alpha}$ denotes the covariant derivative, and $T_{\alpha \beta}$ is the isotropic energy-momentum tensor. The above equations can be expressed in Einstein tensor as given below:

$$
G_{\alpha \beta}=\frac{\kappa}{F}\left(T_{\alpha \beta}^{m}+T_{\alpha \beta}^{D}\right)
$$


where

$$
\begin{aligned}
T_{\alpha \beta}^{D} & =\frac{1}{\kappa}\left[\frac{f(R)-R F(R)}{2} g_{\alpha \beta}+\nabla_{\alpha} \nabla_{\beta} F(R)\right. \\
& \left.-g_{\alpha \beta} \nabla^{\alpha} \nabla_{\alpha} F(R)\right]
\end{aligned}
$$

represent the action energy-momentum tensor. By considering the comoving coordinates bounded by cylindrically symmetric nonstatic spacetime geometry, which contains the dissipative nature of the fluid defines by interior metric,

$$
d s_{-}{ }^{2}=-A^{2} d t^{2}+B^{2} d r^{2}+C^{2} d \phi^{2}+D^{2} d z^{2} .
$$

Here $A=A(t, r), B=B(t, r), C=C(t, r), D=D(t, r)$ are the metric functions. We labeled the coordinates $x^{0}=t, x^{1}=r$, $x^{2}=\phi$ and $x^{3}=z$.

The tensor $T_{\alpha \beta}^{-}$of the dissipative collapsing matter distribution is

$$
\begin{aligned}
T_{\alpha \beta}^{-}= & (\mu+p+\Pi) V_{\alpha} V_{\beta}+(p+\Pi) g_{\alpha \beta}+q_{\alpha} V_{\beta}+q_{\beta} V_{\alpha} \\
& +\epsilon l_{\alpha} l_{\beta}+\pi_{\alpha \beta},
\end{aligned}
$$

where $\mu$ is the energy density, $p$ is the isotropic pressure, $\Pi$ the bulk viscosity, $q^{\alpha}$ the heat flux, $\pi_{\alpha \beta}$ is the shear viscosity, $\epsilon$ the radiation density, and $V^{\alpha}$ the four velocity of the fluid. Furthermore, $l^{\alpha}$ denotes radial null four vector. The above extents gratify the results as below.

$$
\begin{aligned}
V^{\alpha} V_{\alpha} & =-1, \\
V^{\alpha} q_{\alpha} & =0, \\
l^{\alpha} V_{\alpha} & =-1, \\
l^{\alpha} l_{\alpha} & =0, \\
\pi_{\mu \nu} V^{\nu} & =0, \\
\pi_{[\mu \nu]} & =0, \\
\pi_{\alpha}^{\alpha} & =0 .
\end{aligned}
$$

In the general nonreversible thermodynamics taken into [44, 45],

$$
\begin{gathered}
\pi_{\alpha \beta}=-2 \eta \sigma_{\alpha \beta}, \\
\Pi=-\zeta \Theta ;
\end{gathered}
$$

wherever $\eta$ is the factor of shear viscosity and $\zeta$ denotes the factor of bulk viscosity, $\sigma_{\alpha \beta}$ and $\Theta$ are the shear tensor and heat flow expansion, respectively.

The shear tensor $\sigma_{\alpha \beta}$ is defined by

$$
\sigma_{\alpha \beta}=V_{(\alpha ; \beta)}+a_{\left(\alpha V_{\beta}\right)}-\frac{1}{3} \Theta h_{\alpha \beta},
$$

where the acceleration $a_{\alpha}$ and the expansion $\Theta$ are recognized by

$$
\begin{aligned}
& a_{\alpha}=V_{\alpha ; \beta} V^{\beta}, \\
& \Theta=V_{; \alpha}^{\alpha},
\end{aligned}
$$

and $h_{\alpha \beta}=g_{\alpha \beta}+V_{\alpha} V_{\beta}$ is the projector onto the hypersurface orthogonal to the four velocity. let us define the following quantities for the given metric:

$$
\begin{aligned}
V^{\alpha} & =A^{-1} \delta_{0}^{\alpha}, \\
q^{\alpha} & =q B^{-1} \delta_{1}^{\alpha}, \\
l^{\alpha} & =A^{-1} \delta_{0}^{\alpha}+B^{-1} \delta_{1}^{\alpha},
\end{aligned}
$$

where $q$ depending on $t$ and $r$. Also it trails from (8) so that

$$
\begin{aligned}
& \pi_{0 \alpha}=0, \\
& \pi_{1}^{1}=-2 \pi_{2}^{2}=-2 \pi_{3}^{3} .
\end{aligned}
$$

In a more explicit formation we can write

$$
\pi_{\alpha \beta}=\Omega\left(\chi_{\alpha} \chi_{\beta}-\frac{1}{3} h_{\alpha \beta}\right),
$$

where $\chi^{\alpha}$ is a unit four vector along the radial direction, fulfilling the conditions

$$
\begin{aligned}
\chi^{\alpha} \chi_{\alpha} & =1, \\
\chi^{\alpha} V_{\alpha} & =0, \\
\chi^{\alpha} & =B^{-1} \delta_{1}^{\alpha},
\end{aligned}
$$

and $\Omega=(3 / 2) \pi_{1}^{1}$.

From the information of (13), we obtain the following nonnull components of shear tensor.

$$
\begin{aligned}
& \sigma_{11}=\frac{B^{2}}{3 A}\left[\Sigma_{1}-\Sigma_{3}\right], \\
& \sigma_{22}=\frac{C^{2}}{3 A}\left[\Sigma_{2}-\Sigma_{1}\right], \\
& \sigma_{33}=\frac{D^{2}}{3 A}\left[\Sigma_{3}-\Sigma_{2}\right],
\end{aligned}
$$

where

$$
\sigma_{\alpha \beta} \sigma^{\alpha \beta}=\sigma^{2}=\frac{1}{3 A^{2}}\left[\Sigma_{1}^{2}+\Sigma_{2}^{2}+\Sigma_{3}^{2}\right]
$$

where

$$
\begin{aligned}
& \Sigma_{1}=\frac{\dot{B}}{B}-\frac{\dot{C}}{C}, \\
& \Sigma_{2}=\frac{\dot{C}}{C}-\frac{\dot{D}}{D}, \\
& \Sigma_{3}=\frac{\dot{D}}{D}-\frac{\dot{B}}{B} .
\end{aligned}
$$

Here dot denotes derivative w.r.t. $t$.

By using (12) and (13), we get

$$
\begin{aligned}
& a_{1}=\frac{\dot{A}}{A}, \\
& \Theta=\frac{1}{A}\left(\frac{\dot{B}}{B}+\frac{\dot{C}}{C}+\frac{\dot{D}}{D}\right),
\end{aligned}
$$

where prime represents derivative with respect to $r$. 


\section{Modified Field Equations in $f(R)$ Gravity}

The modified field equation (4) for cylindrical symmetric spacetime in $f(R)$ metric formalism gives the following system of equations.

$$
\begin{aligned}
& \left(\frac{A}{B}\right)^{2}\left[-\frac{C^{\prime \prime}}{C}-\frac{D^{\prime \prime}}{D}+\frac{B^{\prime}}{B}\left(\frac{C^{\prime}}{C}+\frac{D^{\prime}}{D}\right)-\frac{C^{\prime} D^{\prime}}{C D}\right] \\
& +\left(\frac{\dot{B} \dot{C}}{B C}+\frac{\dot{B} \dot{D}}{B D}+\frac{\dot{C} \dot{D}}{C D}\right)=\frac{\kappa}{F}\left[(\mu+\epsilon) A^{2}\right. \\
& +\frac{A^{2}}{\kappa}\left\{-\left(\frac{f(R)-R F(R)}{2}\right)\right. \\
& -\frac{\dot{F}}{A^{2}}\left(\frac{\dot{B}}{B}+\frac{\dot{C}}{C}+\frac{\dot{D}}{D}\right)+\frac{F^{\prime \prime}}{B^{2}} \\
& \left.\left.+\frac{F^{\prime}}{B^{2}}\left(\frac{C^{\prime}}{C}+\frac{D^{\prime}}{D}-\frac{B^{\prime}}{B}\right)\right\}\right] \text {, } \\
& -\left(\frac{\dot{C}^{\prime}}{C}+\frac{\dot{D}^{\prime}}{D}-\frac{\dot{B} C^{\prime}}{B C}-\frac{\dot{B} D^{\prime}}{B D}-\frac{\dot{C} A^{\prime}}{A C}-\frac{\dot{D} A^{\prime}}{A D}\right) \\
& =\frac{\kappa}{F}\left[-(q+\epsilon) A B+\frac{1}{\kappa}\left(\dot{F}^{\prime}-\frac{A^{\prime} \dot{F}}{A}-\frac{\dot{B} F^{\prime}}{B}\right)\right], \\
& -\left(\frac{B}{A}\right)^{2}\left(\frac{\ddot{C}}{C}+\frac{\ddot{D}}{D}+\frac{\dot{C} \dot{D}}{C D}-\frac{\dot{A} \dot{C}}{A C}-\frac{\dot{A} \dot{D}}{A D}\right)+\left(\frac{C^{\prime} D^{\prime}}{C D}\right. \\
& \left.+\frac{A^{\prime} C^{\prime}}{A C}+\frac{A^{\prime} D^{\prime}}{A D}\right)=\frac{\kappa}{F}\left[\left(p+\Pi+\epsilon+2 \frac{\Omega}{3}\right) B^{2}\right. \\
& +\frac{B^{2}}{\kappa}\left\{\left(\frac{f(R)-R F(R)}{2}\right)+\frac{\ddot{F}}{A^{2}}\right. \\
& \left.\left.+\frac{\dot{F}}{A^{2}}\left(\frac{\dot{C}}{C}+\frac{\dot{D}}{D}-\frac{\dot{A}}{A}\right)-\frac{F^{\prime}}{B^{2}}\left(\frac{A^{\prime}}{A}+\frac{C^{\prime}}{C}+\frac{D^{\prime}}{D}\right)\right\}\right] \text {, } \\
& -\left(\frac{C}{A}\right)^{2}\left[\frac{\ddot{B}}{B}+\frac{\ddot{D}}{D}-\frac{\dot{A}}{A}\left(\frac{\dot{B}}{B}+\frac{\dot{D}}{D}\right)+\frac{\dot{B} \dot{D}}{B D}\right]+\left(\frac{C}{B}\right)^{2} \\
& \cdot\left[\frac{A^{\prime \prime}}{A}+\frac{D^{\prime \prime}}{D}-\frac{A^{\prime}}{A}\left(\frac{B^{\prime}}{B}-\frac{D^{\prime}}{D}\right)-\frac{B^{\prime} D^{\prime}}{B D}\right] \\
& =\frac{\kappa}{F}\left[\left(p+\Pi-\frac{\Omega}{3}\right) C^{2}\right. \\
& -\frac{C^{2}}{\kappa}\left\{-\left(\frac{f(R)-R F(R)}{2}\right)-\frac{\ddot{F}}{A^{2}}+\frac{F^{\prime \prime}}{B^{2}}\right. \\
& +\frac{\dot{F}}{A^{2}}\left(\frac{\dot{A}}{A}-\frac{\dot{B}}{B}-\frac{\dot{C}}{C}-\frac{\dot{D}}{D}\right) \\
& \left.\left.+\frac{F^{\prime}}{B^{2}}\left(\frac{A^{\prime}}{A}-\frac{B^{\prime}}{B}+\frac{C^{\prime}}{C}+\frac{D^{\prime}}{D}\right)\right\}\right] \text {, }
\end{aligned}
$$

where $\widetilde{M}(\nu)$ is the total mass and $v$ is the usual retarded time coordinate, while $\gamma$ is a constant having the dimension of 1/length. Moreover, the interior spacetime of manifold $\mathrm{M}^{-}$ over the boundary hypersurface $\Sigma$ is

$$
k_{-}(t, r)=r-r_{\Sigma}=0 .
$$

Here $r_{\Sigma}$ is constant. The interior metric of $M^{-}$over bounding hypersurface becomes

$$
d s_{-}^{2} \stackrel{\Sigma}{=}-d \tau^{2}+C^{2} d \phi^{2}+D^{2} d z^{2}
$$


where

$$
d \tau \stackrel{\Sigma}{=} A d t
$$

indicates the time coordinate on hypersurface $\Sigma$ and $\stackrel{\Sigma}{=}$ means the estimation is applied on $\Sigma$ of both sides.

In a similar manner, we prescribed the exterior lineelement $M^{+}$on $3 D$ hypersurface $\Sigma$ takes the form

$$
k_{+}(\nu, \widetilde{R})=\widetilde{R}-\widetilde{R}_{\Sigma}(\nu)=0
$$

Thus, the exterior spacetime manifold $\mathrm{M}^{+}$on hypersurface is given by

$$
\begin{aligned}
d s_{+}^{2} \stackrel{\Sigma}{=} & -2\left(-\frac{\widetilde{M}(\nu)}{\widetilde{R}}+\frac{d \widetilde{R}_{\Sigma}(\nu)}{d \nu}\right) d \nu^{2} \\
& +\widetilde{R}^{2}\left(d \phi^{2}+\gamma^{2} d z^{2}\right) .
\end{aligned}
$$

The following conditions due to Darmois [50] are to be satisfied.

(i) The continuity of the first fundamental form on $\Sigma$ becomes

$$
d s_{\Sigma}^{2}=\left(d s_{-}^{2}\right)_{\Sigma}=\left(d s_{+}^{2}\right)_{\Sigma}
$$

(ii) The continuity of the second fundamental form over boundary three-surface is as follows:

$$
\left[K_{\alpha \beta}\right]=K_{\alpha \beta}^{+}-K_{\alpha \beta}^{-}=0,
$$

where $K_{\alpha \beta}$ is the extrinsic curvature over the hypersurface $\Sigma$ and given into

$$
\begin{aligned}
K_{\alpha \beta}^{ \pm}=-n_{\mu}^{ \pm}\left(\frac{\partial^{2} x_{ \pm}^{\mu}}{\partial \rho^{\alpha} \partial \rho^{\beta}}+\Gamma_{a b}^{\mu} \frac{\partial x_{ \pm}^{a} \partial x_{ \pm}^{b}}{\partial \rho^{\alpha} \partial \rho^{\beta}}\right), & \\
& (\mu, a, b=0,1,2,3) .
\end{aligned}
$$

From the above equation of the extrinsic curvature, the Christoffel symbols are calculated for the interior and exterior manifolds $M^{-}$and $M^{+}$accordingly and we labeled $\left(\rho^{0}, \rho^{2}, \rho^{3}\right)=(\tau, \phi, z)$ for the intrinsic coordinates on boundary surface $\Sigma$ and $n_{\mu}^{ \pm}$are the components of the outward unit normal over $\Sigma$ in the coordinates $x_{ \pm}^{\mu}$, thus we have

$$
\begin{aligned}
& n_{\mu}^{-} \stackrel{\Sigma}{=}(0, B, 0,0), \\
& n_{\mu}^{+} \stackrel{\Sigma}{=} \lambda\left(-\frac{d \widetilde{R}}{d \nu}, 1,0,0\right), \\
& \lambda=\frac{1}{2\left(-\widetilde{M}(\nu) / \widetilde{R}+d \widetilde{R}_{\Sigma}(\nu) / d \nu\right)^{1 / 2}} .
\end{aligned}
$$

The following expressions are taken from the continuity of the first fundamental form:

$$
\begin{gathered}
C\left(t, \widetilde{r}_{\Sigma}\right) \stackrel{\Sigma}{=} \widetilde{R}_{\Sigma}(\nu), \\
D\left(t, \widetilde{r}_{\Sigma}\right) \stackrel{\Sigma}{=} \gamma \widetilde{R}_{\Sigma}(\nu), \\
\frac{d t}{d \tau}=\frac{1}{A}, \\
\frac{d \nu}{d \tau}=\lambda .
\end{gathered}
$$

Hence, the nonzero components of extrinsic curvature $K_{\alpha \beta}^{ \pm}$ are

$$
\begin{aligned}
& K_{00}^{-}=\left(-\frac{A^{\prime}}{A B}\right)_{\Sigma}, \\
& K_{00}^{+}=\left[\left(\frac{d v}{d \tau}\right)^{-1}\left(\frac{d^{2} v}{d \tau^{2}}\right)-\frac{\widetilde{M}}{\widetilde{R}^{2}}\left(\frac{d v}{d \tau}\right)\right]_{\Sigma}, \\
& K_{22}^{-}=\left(\frac{C^{\prime} C}{B}\right)_{\Sigma}, \\
& K_{33}^{-}=\left(\frac{D^{\prime} D}{B}\right)_{\Sigma}, \\
& K_{22}^{+}=\left[\widetilde{R}\left(\frac{d \widetilde{R}}{d \tau}\right)-2 \widetilde{M}\left(\frac{d v}{d \tau}\right)\right]_{\Sigma}=\gamma^{-2} K_{33}^{+} .
\end{aligned}
$$

Thus, continuity of the extrinsic curvature is organized with (38); we obtain the following results over hypersurface $\Sigma[16$, 51]:

$$
\begin{gathered}
\widetilde{M}(\nu) \stackrel{\Sigma}{=} \frac{\widetilde{R}}{2}\left[\left(\frac{\widetilde{R}}{A}\right)^{2}-\left(\frac{\widetilde{R}^{\prime}}{B}\right)^{2}\right], \\
E \stackrel{\Sigma}{=} \frac{l}{8}+\gamma \widetilde{M}(\nu), \\
q \stackrel{\Sigma}{=}\left(P+\Pi+\frac{2 \Omega}{3}\right)+\frac{T_{01}^{D}}{A B}+\frac{T_{11}^{D}}{B^{2}} .
\end{gathered}
$$

Here $T_{01}^{D}$ and $T_{11}^{D}$ are the dark source components given in Appendices (A.1) and (A.2), respectively.

Therefore, (44) shows the total mass of the interior boundary surface $\Sigma$, whereas (45) gives the smooth association between the $C$ energy for the cylindrical symmetric geometry and the active mass on hypersurface $\Sigma$. Moreover, (46) describes the linear association among the fluid quantities $(P, \Pi, \Omega, q)$ over the boundary three-surface $\Sigma$. Thus effective pressure is in usual nonvanishing over $\Sigma$ due to dissipative nature of the dark source and this dissipation through viscosity parameters in the form of heat flow and undergoes free radiation streaming on the boundary surface. The extra components on the right hand side defines the dark source and appeared due to higher curvature of the geometry. It should be very interesting to mention that if the fluid has 
no dark source components then effective pressure and heat flux are equal on the boundary surface $\Sigma$.

Furthermore, other important results of the collapsing fluid are performed on the bounding three-surface $\Sigma$ for the matching conditions of the Ricci invariant $R$ and its normal derivative. The detailed description of matching conditions in $f(R)$ gravity theory has been given in $[50,52-56]$. We require the matching conditions for the continuity of both spacetimes $M^{-}$and $M^{+}$over the hypersurface $\Sigma$; the following matching conditions $f(R)$ theory are given by

$$
\begin{aligned}
\left.R\right|^{ \pm} & =0, \\
f_{, R R}\left[\left.\partial_{\gamma} R\right|^{ \pm}\right. & =0, \\
f_{, R R} & \neq 0 .
\end{aligned}
$$

The above expressions are required for the continuity of Ricci invariant $R$ over the boundary surface $\Sigma$ in the cylindrical collapsing fluid.

3.2. Dynamical Equations. The proper time and radial derivatives are given; we use Misner and Sharp technique [2].

$$
\begin{aligned}
& D_{T}=\frac{1}{A} \frac{\partial}{\partial t}, \\
& D_{C}=\frac{1}{C^{\prime}} \frac{\partial}{\partial r},
\end{aligned}
$$

where $C$ is the areal radius of a spherical surface inside the limit. The velocity of the collapsing matter is described by the proper time derivative of $C$ and $D$ [17]; i.e.,

$$
\begin{aligned}
& U=D_{T} C=\frac{\dot{C}}{A}, \\
& V=D_{T} D=\frac{\dot{D}}{A},
\end{aligned}
$$

which is always negative. Applying these results and (27), it turns out into

$$
\begin{aligned}
\widetilde{E} & \equiv \frac{C^{\prime}}{B} \\
& =\left[\left(\frac{V C+U D}{D}\right)^{2}-\frac{8}{D}\left(m(r, t)-\frac{l}{8}\right)\right]^{1 / 2}-\frac{C D^{\prime}}{B D} .
\end{aligned}
$$

The rate of change of mass is w.r.t. proper time in (27); we use (21), (22), and (23)-(25) as follows:

$$
\begin{aligned}
& D_{T} m(r, t)=\frac{C D}{F}\left[-4 \pi\left\{\left(\mu+2 \epsilon-p-\Pi+\frac{4 \Omega}{3}\right) U\right.\right. \\
& +\widetilde{E}(q+\epsilon)\}
\end{aligned}
$$

$$
\begin{aligned}
& -U\left\{\frac{\widetilde{E}^{2}}{C^{\prime 2}}\left(\frac{F^{\prime} C^{\prime}}{C}+\frac{F^{\prime} D^{\prime}}{D}-\frac{3 \widetilde{E} F^{\prime} D_{C} B}{2}\right)\right. \\
& +\frac{\widetilde{E}^{2}}{2}\left(\frac{D_{C} A D_{T} F}{\dot{C}}+\frac{3 F^{\prime \prime}}{C^{\prime 2}}\right) \\
& +\frac{\widetilde{E}^{2} F}{D}\left(D_{C C} D+\frac{\left(D_{C} D\right)^{2}}{4 D}+\frac{D_{C} D}{4 C}\right) \\
& +\frac{\widetilde{E}^{2} F D_{C C} C}{C}-\frac{\widetilde{E}^{3} F D_{C} B}{C^{\prime}}\left(\frac{1}{C}+\frac{D_{C} D}{D}\right) \\
& -\frac{F}{B}\left(\frac{V D_{T} B}{D}+D_{T T} B\right)-\frac{F}{4}\left(\frac{D_{T T} C}{C}+\frac{D_{T T} D}{D}\right) \\
& \left.+\frac{R F(R)}{2}-\frac{f(R)}{2}\right\}-U^{3}\left\{\frac{\dot{F}}{\dot{C}}\left(\frac{D_{T} A}{2 \dot{C}}-\frac{1}{C}\right)\right. \\
& \left.-\frac{\ddot{F}}{2 \dot{C}^{2}}+\frac{F D_{T} A}{4 C \dot{C}}\right\} \\
& +U^{2}\left\{\frac{\widetilde{E}^{2}}{\dot{C}}\left(\frac{3 \dot{F} D_{T} B}{2 \widetilde{E} C^{\prime}}-\frac{F^{\prime} D_{C} A}{2 C^{\prime}}\right)+\frac{V \dot{F}}{\dot{C} D}\right. \\
& +\frac{F D_{T} B}{B}\left(\frac{1}{C}-\frac{D_{T} A}{\dot{C}}\right) \\
& -\frac{\widetilde{E}^{2} F}{\dot{C}}\left(D_{\mathrm{CC}} A-\frac{\widetilde{E} D_{C} A D_{C} B}{C^{\prime}}+\frac{D_{C} A}{2 C}\right) \\
& \left.+\frac{V F}{2 D}\left(\frac{3}{4 C}-\frac{D_{T} A}{\dot{C}}\right)\right\}-\frac{V^{2} F}{2 D}\left(\frac{\widetilde{E}^{2} D_{C} A}{\dot{D}}-\frac{U}{2 D}\right) \\
& -\frac{V^{3} F}{4 D^{2}}\left(\frac{C}{D}+\frac{C D_{T} A}{\dot{D}}\right)+\frac{V F}{4 D}\left\{\frac{C D_{T T} D}{D}+D_{T T} C\right. \\
& \left.+\widetilde{E}^{2}\left(\frac{C\left(D_{C} D\right)^{2}}{2 D^{2}}-\frac{1}{2 C}\right)\right\} \\
& -\frac{\widetilde{E}^{2}}{2 C^{\prime}}\left\{\widetilde{E} D_{T} B\left(D_{C} F+\frac{F}{2 C}-\frac{C\left(D_{C} D\right)^{2} F}{2 D^{2}}\right)\right. \\
& -F\left(\frac{D_{T} C^{\prime}}{2 C}+\frac{D_{T} D^{\prime}}{2 D}\right) \\
& \left.\left.+\frac{F D_{C} D}{2 D}\left(\frac{C D_{T} D^{\prime}}{D}+D_{T} C^{\prime}\right)-D_{T} F^{\prime}\right\}\right] \text {. }
\end{aligned}
$$

The overhead result leads to telling us that the variation rate of the total energy interior of the cylinder of radius $\mathrm{C}$ and the R.H.S of (51), $(\mu+2 \epsilon-p-\Pi+4 \Omega / 3) U$ reflects that the energy is increasing in the interior surface of radius $C$ (in case of collapsing situation $(U<0))$ through the rate of work being done by the active radial pressure, the energy density $\mu$, and the radiation pressure $\epsilon$. The usual thermodynamical result is $\pi_{\alpha \beta}=-2 \eta \sigma_{\alpha \beta}$ used in the relaxation phase. The second value $\widetilde{E}(q+\epsilon)$ of the right-hand side illustrates the matter energy of the fluid, which is leaving the cylindrical surface. Moreover, 
all other extra terms play the natural role of the dark energy dissipative source under the formation of modified theory of gravity and decreasing the pressure in the core of the star due to continuous collapsing phase of the dark energy. Similarly, we can calculate

$$
\begin{aligned}
& D_{C} m(r, t)=\frac{C D}{2 F}\left[\kappa \left\{\mu+2 \epsilon+p+\Pi+\frac{2 \Omega}{3}+\frac{U}{\widetilde{E}}(q\right.\right. \\
& +\epsilon)\}+\frac{U^{3} F D_{C} A}{2 C \dot{C}}+U^{2}\left\{\frac{D_{T} F D_{C} A}{\dot{C}}+\frac{F}{C}\left(\frac{D_{C} D}{4 D}\right.\right. \\
& \left.\left.-\frac{D_{T} A}{\dot{C}}\right)\right\}-U\left\{\widetilde{E} D_{C} F\left(\frac{\widetilde{E} D_{C} A}{\dot{C}}-\frac{D_{T} B}{C^{\prime}}\right)\right. \\
& +\frac{D_{T} F D_{T} A}{\dot{C}} \\
& +F\left(\frac{1}{C^{\prime}}\left(\frac{D_{T} C^{\prime}}{2 C}+\frac{D_{T} D^{\prime}}{2 D}-\frac{\tilde{E} D_{T} B D_{C} D}{D}\right)\right. \\
& \left.\left.+\frac{\widetilde{E}^{2}}{\dot{C}}\left(\frac{D_{C} A}{C}+\frac{D_{C} A D_{C} D}{D}\right)\right)\right\} \\
& -\frac{V^{2} F}{2 D^{2}}\left\{C\left(\frac{U D_{C} A}{\dot{C}}+\frac{D_{C} D}{2 D}\right)-1\right\} \\
& +\frac{V F}{2 C^{\prime} D}\left(\frac{C D_{T} D^{\prime}}{D}+D_{T} C^{\prime}\right)+\frac{U V F}{D}\left(\frac{1}{2 C}\right. \\
& \left.-\frac{D_{T} A}{\dot{C}}\right)-\widetilde{E}^{2} F\left\{\frac { D _ { C } D } { 2 D ^ { 2 } } \left(C D_{C C} D+D_{C} D\right.\right.
\end{aligned}
$$

$$
\begin{aligned}
& \left.-\frac{C\left(D_{C} D\right)^{2}}{2 D}-\frac{\widetilde{E} C D_{C} B D_{C} D}{C^{\prime}}\right)-\frac{1}{2 C}\left(D_{C C} C\right. \\
& \left.-\frac{3 D_{C} D}{2 D}-\frac{\widetilde{E} D_{C} B}{C^{\prime}}\right)+\frac{1}{2 D}\left(D_{C} D D_{C C} C\right. \\
& \left.\left.-D_{C C} D\right)\right\}-\frac{\widetilde{E}}{C^{\prime}}\left(D_{T} F D_{T} B+\widetilde{E}^{2} D_{C} F D_{C} B\right. \\
& \left.+\frac{F D_{T} B D_{T} D}{D}\right)+D_{T T} F+\frac{F^{\prime \prime} \widetilde{E}^{2}}{C^{\prime 2}}-\frac{U D_{T} F^{\prime}}{C^{\prime}} \\
& \left.+\frac{F D_{T T} C}{C}+\frac{F D_{T T} D}{D}\right] .
\end{aligned}
$$

This solution of (52) explains how variational quantities affect the matter distribution between the neighboring exteriors in the object of radius $\mathrm{C}$. The picture of the first two quantities on the R.H.S of (52) is $\mu+2 \epsilon$ related with energy density of the fluid and plus the addiction of the null fluid conferring dissipation in the outgoing streaming approximation. The last factor $(U / \widetilde{E})(q+\epsilon)$ plays negative role in the collapsing situation and estimates the dissipation in terms of heat flow and radiation streaming. The extra values signify the input of the dark energy (DE) due to its higher order of the curvature matter. We Apply integration on (52) with $C$; we get

$$
\begin{aligned}
& m(r, t)=\frac{1}{2} \int_{0}^{C} \frac{C D}{2 F}\left[\kappa\left\{\mu+2 \epsilon+p+\Pi+\frac{2 \Omega}{3}+\frac{U}{\widetilde{E}}(q+\epsilon)\right\}+\frac{U^{3} F D_{C} A}{2 C \dot{C}}+U^{2}\left\{\frac{D_{T} F D_{C} A}{\dot{C}}+\frac{F}{C}\left(\frac{D_{C} D}{4 D}-\frac{D_{T} A}{\dot{C}}\right)\right\}\right. \\
& -U\left\{\widetilde{E} D_{C} F\left(\frac{\widetilde{E} D_{C} A}{\dot{C}}-\frac{D_{T} B}{C^{\prime}}\right)+\frac{D_{T} F D_{T} A}{\dot{C}}\right. \\
& \left.+F\left(\frac{1}{C^{\prime}}\left(\frac{D_{T} C^{\prime}}{2 C}+\frac{D_{T} D^{\prime}}{2 D}-\frac{\widetilde{E} D_{T} B D_{C} D}{D}\right)+\frac{\widetilde{E}^{2}}{\dot{C}}\left(\frac{D_{C} A}{C}+\frac{D_{C} A D_{C} D}{D}\right)\right)\right\}-\frac{V^{2} F}{2 D^{2}}\left\{C\left(\frac{U D_{C} A}{C}+\frac{D_{C} D}{2 D}\right)-1\right\} \\
& +\frac{V F}{2 C^{\prime} D}\left(\frac{C D_{T} D^{\prime}}{D}+D_{T} C^{\prime}\right)+\frac{U V F}{D}\left(\frac{1}{2 C}-\frac{D_{T} A}{\dot{C}}\right) \\
& +\widetilde{E}^{2} F\left\{\frac{D_{C} D}{2 D^{2}}\left(C D_{C C} D+D_{C} D-\frac{C\left(D_{C} D\right)^{2}}{2 D}-\frac{\widetilde{E} C D_{C} B D_{C} D}{C^{\prime}}\right)-\frac{1}{2 C}\left(D_{C C} C-\frac{3 D_{C} D}{2 D}-\frac{\widetilde{E D}_{C} B}{C^{\prime}}\right)\right. \\
& \left.+\frac{1}{2 D}\left(D_{C} D D_{C C} C-D_{C C} D\right)\right\}-\frac{\widetilde{E}}{C^{\prime}}\left(D_{T} F D_{T} B+\widetilde{E}^{2} D_{C} F D_{C} B+\frac{F D_{T} B D_{T} D}{D}\right)+D_{T T} F+\frac{F^{\prime \prime} \widetilde{E}^{2}}{C^{\prime 2}}-\frac{U D_{T} F^{\prime}}{C^{\prime}}
\end{aligned}
$$


The above equation gives the total mass in terms of C-energy inside the cylinder with the contribution of $f(R)$ dark source term. To observe the dynamical equation by assuming Misner and Sharp approach $[2,57]$, the contracted Bianchi identities take the form,

$$
\begin{aligned}
& \left(T_{\alpha \beta}^{(m)}+T_{\alpha \beta}^{(D)}\right)_{; \beta} V_{\alpha}=0, \\
& \left(T_{\alpha \beta}^{(m)}+T_{\alpha \beta}^{(D)}\right)_{; \beta} \chi_{\alpha}=0,
\end{aligned}
$$

which produce

$$
\begin{aligned}
& \frac{1}{A}\left[(\mu+\epsilon)+\left(\mu+p+\Pi+2 \epsilon+\frac{2 \Omega}{3}\right) \frac{\dot{B}}{B}\right. \\
& \left.\quad+\left(\mu+p+\Pi+\epsilon-\frac{\Omega}{3}\right)\left(\frac{\dot{C}}{C}+\frac{\dot{D}}{D}\right)\right] \\
& \quad+\frac{1}{B}\left[(q+\epsilon)^{\prime}+(q+\epsilon)\left(\frac{2 A^{\prime}}{A}+\frac{(C D)^{\prime}}{C D}\right)\right]-D_{1} \\
& \quad=0, \\
& \frac{1}{A}\left[(q+\epsilon)+(q+\epsilon)\left(2 \frac{\dot{B}}{B}+\frac{\dot{C}}{C}+\frac{\dot{D}}{D}\right)\right] \\
& \quad+\frac{1}{B}\left[\left(p+\Pi+\epsilon+\frac{2 \Omega}{3}\right)^{\prime}\right. \\
& \left.\quad+\left(\mu+p+\Pi+2 \epsilon+\frac{2 \Omega}{3}\right)^{\prime} \frac{A^{\prime}}{A}+(\epsilon+\Omega) \frac{(C D)^{\prime}}{C D}\right] \\
& +D_{2}=0 .
\end{aligned}
$$

Here $D_{1}$ and $D_{2}$ are the dark source components shown in Appendix (A.3) and (A.4), respectively.

From (23) and (48)-(50), it follows that

$$
\begin{aligned}
& D_{T}(\mu+\epsilon)+\frac{1}{3}(3 \mu+3 p+3 \Pi+4 \epsilon) \Theta \\
& +\frac{1}{3}(\epsilon+\Omega)\left(\frac{2 \dot{B}}{A B}-\frac{U}{C}-\frac{V}{D}\right)+\widetilde{E} D_{C}(q+\epsilon) \\
& \quad+(q+\epsilon)\left(2 \frac{a_{1}}{B}+\frac{\widetilde{E}}{C}+\frac{D^{\prime}}{B D}\right)-D_{1}=0 . \\
& D_{T}(q+\epsilon)+(q+\epsilon)\left(\Theta+\frac{D_{T} B}{B}\right) \\
& +\widetilde{E} D_{C}\left(p+\Pi+\epsilon+\frac{2 \Omega}{3}\right) \\
& \quad+\left(\mu+p+\Pi+2 \epsilon+\frac{2 \Omega}{3}\right) \frac{a_{1}}{B}+(\epsilon+\Omega) \frac{(C D)^{\prime}}{B C D} \\
& \quad+D_{2}=0 .
\end{aligned}
$$

The acceleration $D_{T} U$ of the dissipative viscous collapsing source is attained by using (23) and (48)-(50); it becomes

$$
\begin{aligned}
D_{T} U & =-\frac{m(r, t)}{C D}+\frac{l}{8 C D}-\frac{4 \pi C}{F}\left(p+\Pi+\epsilon+\frac{2 \Omega}{3}\right) \\
+ & \left(\frac{\widetilde{E}}{2}+\frac{D^{\prime} C}{2 B D}\right) \frac{a_{1}}{B}-\frac{C}{F}\left[\frac{D_{T T} F}{2}\right. \\
+ & \frac{U D_{T} F}{2}\left(\frac{1}{C}-\frac{D_{T} A}{\dot{C}}\right)+\frac{V D_{T} F}{2 D}-\frac{\widetilde{E} D^{\prime} D_{C} F}{2 B D} \\
- & \left.\frac{\widetilde{E}^{2} D_{C} F}{2}\left(\frac{U D_{C} A}{C^{2}}+\frac{1}{C}\right)\right]+\frac{D_{T T} C}{2}-\frac{C D_{T T} D}{2 D} \\
- & \frac{D_{T} A}{2 A}\left(U-\frac{C V}{D}\right)-\frac{1}{4 D}\left(U V-\frac{\widetilde{E} D^{\prime}}{B}\right) \\
+ & \frac{1}{8 D^{2}}\left(C V^{2}-\frac{C D^{\prime 2}}{B^{2}}\right)+\frac{1}{8 C}\left(U^{2}-\widetilde{E}^{2}\right) \\
- & \frac{C(f(R)-R F(R))}{4 F} .
\end{aligned}
$$

After, inserting $a_{1} / B$ from (59) into (58), we get

$$
\begin{aligned}
& \left(\mu+p+\Pi+2 \epsilon+\frac{2 \Omega}{3}\right) D_{T} U=-(\mu+p+\Pi+2 \epsilon \\
& \left.+\frac{2 \Omega}{3}\right)\left[\frac{m(r, t)}{C D}-\frac{l}{8 C D}\right. \\
& +\frac{4 \pi C}{F}\left(p+\Pi+\epsilon+\frac{2 \Omega}{3}\right)+\frac{C(f(R)-R F(R))}{4 F} \\
& +\frac{C D_{T T} F}{2 F}+\left(\frac{1}{C}-\frac{D_{T} A}{\dot{C}}\right) \frac{U C D_{T} F}{2 F}+\frac{V C D_{T} F}{2 D F} \\
& -\frac{\widetilde{E} C D^{\prime} D_{C} F}{2 B D F}-\frac{\widetilde{E}^{2} C D_{C} F}{2 F}\left(\frac{1}{C}+\frac{U D_{C} A}{\dot{C}}\right) \\
& -\frac{D_{T T} C}{2}+\frac{C D_{T T} D}{2 D}+\frac{D_{T} A}{2 A}\left(U-\frac{V C}{D}\right) \\
& +\frac{1}{4 D}\left(U V-\frac{\widetilde{E} D^{\prime}}{B}\right)-\frac{1}{8 D^{2}}\left(V^{2} C-\frac{C D^{\prime 2}}{B^{2}}\right) \\
& \left.-\frac{1}{8 C}\left(U^{2}-\widetilde{E}^{2}\right)\right]-\left(\frac{\widetilde{E}}{2}+\frac{D^{\prime} C}{2 B D}\right) \\
& \cdot\left[\widetilde{E} D_{C}\left(p+\Pi+\epsilon+\frac{2 \Omega}{3}\right)+\frac{\widetilde{E}}{C}(\epsilon+\Omega)\right. \\
& \left.+\frac{D^{\prime}}{B D}(\epsilon+\Omega)\right]-\left(\frac{\widetilde{E}}{2}+\frac{D^{\prime} C}{2 B D}\right)\left[D_{T}(q+\epsilon)\right. \\
& \left.+(q+\epsilon)\left(\Theta+\frac{D_{T} B}{B}\right)+D_{2}\right] \text {. }
\end{aligned}
$$

In this interpretation, to analyze the factor $(\mu+p+\Pi+$ $2 \epsilon+2 \Omega / 3$ ), that comes on the L.H.S and on the R.H.S, this 
is effective inertial mass and defines through equivalence principle. It can also be recognized as passive gravitational mass. On the R.H.S, the first term of square bracket illustrates the impacts of the collapsing variables on the active gravitational mass for the cylindrical collapsing object with the dissipative dark source in $\mathrm{f}(\mathrm{R})$ metric theory; this datum was early pointed by Herrera et al. [35] in the context of GR. Moreover, in the second square bracket there is the gradient of the whole active pressure which is influenced by collapsing variables and radiating density. The final bracket includes unalike quantities that define the collapsing nature of the source. The second value of this bracket $(q+\epsilon)$ is positive concluding that dissipation is continuous in the form of heat flow and free streaming radiation and finally decreases the total energy of the system, which reduces the degree of collapse. The last term of this bracket expresses the dark energy source of the dissipative gravitating collapse.

\section{Transport Equations}

The purpose of this profile is to discuss the full causal technique for the viscous dissipative gravitating collapse in self-gravitating system accompanied by heat transference. This study tells us that all collapsing variables should satisfy the transportation equations attained from causal thermodynamics. Therefore, we take the transportation equations for heat, bulk, and shear viscosity from Müller-Israel-Stewart formalism [41-43] for dissipative source. Herrera et al. [35] discussed transportation equations for heat, bulk, and shear viscosity. The entropy flux is given by

$$
\begin{aligned}
S^{\mu}= & S n V^{\mu}+\frac{q^{\mu}}{T}-\left(\beta_{0} \Pi^{2}+\beta_{1} q_{\nu} q^{\nu}+\beta_{2} \pi_{\nu \kappa} \pi^{\nu \kappa}\right) \frac{V^{\mu}}{2 T} \\
& +\frac{\alpha_{0} \Pi q^{\mu}}{T}+\frac{\alpha_{1} \pi^{\mu \nu} q_{v}}{T},
\end{aligned}
$$

where $\beta_{1}$ and $\beta_{2}$ are thermodynamic factors for unalike additions to entropy density, $\alpha_{0}$ and $\alpha_{1}$ are thermodynamics heat coupling factors, and $T$ is temperature.

Furthermore, from the Gibbs equation and Bianchi identities, it follows that

$$
\begin{aligned}
& T S_{; \alpha}^{\alpha}=-\Pi\left[V_{; \alpha}^{\alpha}-\alpha_{0} q_{; \alpha}^{\alpha}+\beta_{0} \Pi_{; \alpha} V^{\alpha}\right. \\
& \left.\quad+\frac{T}{2}\left(\frac{\beta_{0}}{T} V^{\alpha}\right)_{; \alpha} \Pi\right]-q^{\alpha}\left[h_{\alpha}^{\mu}(\ln T)_{, \mu}\left(1+\alpha_{0} \Pi\right)\right. \\
& \quad+V_{\alpha ; \mu} V^{\mu}-\alpha_{0} \Pi_{; \alpha}-\alpha_{1} \pi_{\alpha ; \mu}^{\mu}+\alpha_{1} \pi_{\alpha}^{\mu} h_{\mu}^{\beta}(\ln T)_{, \beta} \\
& \left.\quad+\beta_{1} q_{\alpha ; \mu} V^{\mu}+\frac{T}{2}\left(\frac{\beta_{1}}{T} V^{\mu}\right)_{; \mu} q_{\alpha}\right]-\pi^{\alpha \mu}\left[\sigma_{\alpha \mu}\right. \\
& \left.\quad-\alpha_{1} q_{\mu ; \alpha}+\beta_{2} \pi_{\alpha \mu ; \nu} V^{\nu}+\frac{T}{2}\left(\frac{\beta_{2}}{T} V^{\nu}\right)_{; \nu} \pi_{\alpha \mu}\right]
\end{aligned}
$$

Finally, by the standard procedure, the constitutive transport equations follow from the requirement $S_{; \alpha}^{\alpha} \geq 0$ :

$$
\begin{aligned}
& \tau_{0} \Pi_{, \alpha} V^{\alpha}+\Pi=-\zeta \theta+\alpha_{0} \zeta q_{; \alpha}^{\alpha}-\frac{1}{2} \zeta T\left(\frac{\tau_{0}}{\zeta T} V^{\alpha}\right)_{; \alpha} \Pi . \\
& \tau_{1} h_{\alpha}^{\beta} q_{\beta ; \mu} V^{\mu}+q_{\alpha}=-\kappa\left[h_{\alpha}^{\beta} T_{, \beta}\left(1+\alpha_{0} \Pi\right)+\alpha_{1} \pi_{\alpha}^{\mu} h_{\mu}^{\beta} T_{, \beta}\right. \\
& \left.\quad+T\left(a_{\alpha}-\alpha_{0} \Pi_{; \alpha}-\alpha_{1} \pi_{\alpha ; \mu}^{\mu}\right)\right]-\frac{1}{2} \kappa T^{2}\left(\frac{\tau_{1}}{\kappa T^{2}} V^{\beta}\right)_{; \beta} \\
& \cdot q_{\alpha} \\
& \tau_{2} h_{\alpha}^{\mu} h_{\beta}^{\nu} \pi_{\mu \nu ; \rho} V^{\rho}+\pi_{\alpha \beta}=-2 \eta \sigma_{\alpha \beta}+2 \eta \alpha_{1} q_{\langle\beta ; \alpha\rangle} \\
& \quad \eta T\left(\frac{\tau_{2}}{2 \eta T} V^{\nu}\right)_{; \nu} \pi_{\alpha \beta},
\end{aligned}
$$

with

$$
q_{\langle\beta ; \alpha\rangle}=h_{\beta}^{\mu} h_{\alpha}^{\nu}\left(\frac{1}{2}\left(q_{\mu ; \nu}+q_{\nu ; \mu}\right)-\frac{1}{3} q_{\sigma ; \kappa} h^{\sigma \kappa} h_{\mu \nu}\right)
$$

and where the relaxational times are given by

$$
\begin{aligned}
& \tau_{0}=\zeta \beta_{0}, \\
& \tau_{1}=\kappa T \beta_{1}, \\
& \tau_{2}=2 \eta \beta_{2},
\end{aligned}
$$

where $\zeta$ and $\eta$ are the factors of bulk and shear viscosity. We use the interior metric of the cylindrical structure from (63)(65), to get the following set of equations:

$$
\begin{aligned}
\tau_{0} \dot{\Pi} & =-\left(\zeta+\frac{\tau_{0}}{2} \Pi\right) A \Theta+\frac{A}{B} \alpha_{0} \zeta\left[q^{\prime}+q\left(\frac{A^{\prime}}{A}+\frac{C^{\prime}}{C}\right.\right. \\
+ & \left.\left.\frac{D^{\prime}}{D}\right)\right]-\Pi\left[\frac{\zeta T}{2}\left(\frac{\tau_{0}}{\zeta T}\right)+A\right], \\
\tau_{1} \dot{q} & =-\frac{A}{B} \kappa\left\{T^{\prime}\left(1+\alpha_{0} \Pi+\frac{2}{3} \alpha_{1} \Omega\right)+T\left[\frac{A^{\prime}}{A}-\alpha_{0} \Pi^{\prime}\right.\right. \\
& \left.\left.-\frac{2}{3} \alpha_{1}\left(\Omega^{\prime}+\frac{A^{\prime}}{A} \Omega+\frac{3}{2}\left(\frac{C^{\prime}}{C}+\frac{D^{\prime}}{D}\right) \Omega\right)\right]\right\} \\
& -q\left[\frac{\kappa T^{2}}{2}\left(\frac{\dot{\tau_{1}}}{\kappa T^{2}}\right)+\frac{\tau_{1}}{2} A \Theta+A\right], \\
\tau_{2} \dot{\Omega} & =-\eta\left(\frac{2 \dot{B}}{B}-\frac{\dot{C}}{C}-\frac{\dot{D}}{D}\right)+2 \eta \alpha_{1} \frac{A}{B} q^{\prime} \\
& -\Omega\left[\eta T\left(\frac{\dot{\tau_{2}}}{2 \eta T}\right)+\frac{\tau_{2}}{2} A \Theta+A\right]
\end{aligned}
$$


where we have to analyze the effect on several dissipative variables for the cylindrical interior surface. For this persistence, we use (69) and (60) and get

$$
\begin{aligned}
& \left(\mu+p+\Pi+2 \epsilon+\frac{2}{3} \Omega\right)(1-\Lambda) D_{T} U=(1-\Lambda) F_{\text {grav }} \\
& +F_{\text {hyd }}+\widetilde{E}\left(\frac{\widetilde{E}}{2}+\frac{D^{\prime} C}{2 B D}\right) \\
& +\frac{\kappa}{\tau_{1}}\left\{D_{C^{\prime} T}\left(1+\alpha_{0} \Pi+\frac{2}{3} \alpha_{1} \Omega\right)\right. \\
& \left.-T\left[\alpha_{0} D_{C} \Pi+\frac{2}{3} \alpha_{1}\left(D_{C} \Omega+\frac{3(C D)^{\prime}}{2 C D C^{\prime}} \Omega\right)\right]\right\} \\
& +\left(\frac{\widetilde{E}}{2}+\frac{D^{\prime} C}{2 B D}\right)\left[\frac{\kappa T^{2} q}{2 \tau_{1}} D_{T}\left(\frac{\tau_{1}}{\kappa T^{2}}\right)-D_{T} \epsilon\right]-\left(\frac{\widetilde{E}}{2}\right. \\
& \left.+\frac{D^{\prime} C}{2 B D}\right)\left[\left(\frac{q}{2}+2 \epsilon\right) \Theta-\frac{q}{\tau_{1}}+(q+\epsilon) \frac{D_{T} B}{B}+D_{2}\right] .
\end{aligned}
$$

Here $F_{\text {grav }}$ and $F_{h y d}$ are introduced into the form

$$
\begin{aligned}
F_{\text {grav }} & =-\left(\mu+p+\Pi+2 \epsilon+\frac{2 \Omega}{3}\right)\left[m(r, t)-\frac{l}{8}\right. \\
& +4 \pi\left(p+\Pi+\epsilon+\frac{2 \Omega}{3}\right) \frac{C^{2} D}{F} \\
& +\frac{C^{2} D(f(R)-R F(R))}{4 F}+\frac{C^{2} D D_{T T} F}{2 F} \\
& +\frac{U C^{2} D D_{T} F}{2 F}\left(\frac{1}{C}-\frac{D_{T} A}{\dot{C}}\right)+\frac{V C^{2} D_{T} F}{2 F} \\
& -\frac{\widetilde{E} C^{2} D^{\prime} D_{C} F}{2 B F}-\frac{\widetilde{E}^{2} C^{2} D D_{C} F}{2 F}\left(\frac{1}{C}+\frac{U D_{C} A}{\dot{C}}\right) \\
& -\frac{C D D_{T T} C}{2}+\frac{C^{2} D_{T T} D}{2}+\frac{C D D_{T} A}{2 A}\left(U-\frac{V C}{D}\right) \\
& +\frac{C}{4}\left(U V-\frac{\widetilde{E} D^{\prime}}{B}\right)-\frac{C}{8 D}\left(V^{2} C-\frac{C D^{\prime 2}}{B^{2}}\right) \\
& \left.+-\frac{D}{8}\left(U^{2}-\widetilde{E}^{2}\right)\right] \frac{1}{C D}, \\
F_{h y d} & =-\left(\frac{\widetilde{E}}{2}+\frac{D^{\prime} C}{2 B D}\right)\left[\widetilde{E} D_{C}\left(p+\Pi+\epsilon+\frac{2 \Omega}{3}\right)\right. \\
+ & \left.\frac{\widetilde{E}}{C}(\epsilon+\Omega)+\frac{D^{\prime}}{B D}(\epsilon+\Omega)\right],
\end{aligned}
$$

and $\Lambda$ is defined as

$$
\Lambda=\frac{\kappa T}{\tau_{1}}\left(\mu+p+\Pi+2 \epsilon+\frac{2}{3} \Omega\right)^{-1}\left(1-\frac{2}{3} \alpha_{1} \Omega\right) .
$$

From (68) and (71) subsequently it becomes

$$
\begin{aligned}
& \left(\mu+p+\Pi+2 \epsilon+\frac{2}{3} \Omega\right)(1-\Lambda+\Delta) D_{T} U=(1-\Lambda \\
& +\Delta) F_{\text {grav }}+F_{h y d}+\frac{\widetilde{E} \kappa}{\tau_{1}}\left(\frac{\widetilde{E}}{2}+\frac{D^{\prime} C}{2 B D}\right) \\
& .\left\{D_{C} T\left(1+\alpha_{0} \Pi+\frac{2}{3} \alpha_{1} \Omega\right)\right. \\
& \left.-T\left[\alpha_{0} D_{C} \Pi+\frac{2}{3} \alpha_{1}\left(D_{C} \Omega+\frac{3(C D)^{\prime}}{2 C D C^{\prime}} \Omega\right)\right]\right\} \\
& -\widetilde{E}\left(\frac{\widetilde{E}}{2}+\frac{D^{\prime} C}{2 B D}\right)\left(\mu+p+\Pi+2 \epsilon+\frac{2}{3} \Omega\right) \\
& \cdot \Delta\left(\frac{D_{C} q}{q}+\frac{(C D)^{\prime}}{C D C^{\prime}}\right)+\left(\frac{\widetilde{E}}{2}+\frac{D^{\prime} C}{2 B D}\right) \\
& .\left[\frac{\kappa T^{2} q}{2 \tau_{1}} D_{T}\left(\frac{\tau_{1}}{\kappa T^{2}}\right)-D_{T} \epsilon\right]+\left(\frac{\widetilde{E}}{2}+\frac{D^{\prime} C}{2 B D}\right)\left[\frac{q}{\tau_{1}}\right. \\
& \left.-(q+\epsilon) \frac{D_{T} B}{B}-D_{2}\right]+\left(\frac{\widetilde{E}}{2}+\frac{D^{\prime} C}{2 B D}\right) \frac{\Delta}{\alpha_{0} \zeta q}(\mu \\
& \left.+p+\Pi+2 \epsilon+\frac{2}{3} \Omega\right)\left\{\left[1+\frac{\zeta T}{2} D_{T}\left(\frac{\tau_{0}}{\zeta T}\right)\right] \Pi\right. \\
& \left.+\tau_{0} D_{T} \Pi\right\},
\end{aligned}
$$

where $\Delta$ is recognized as

$$
\Delta=\alpha_{0} \zeta q\left(\mu+p+\Pi+2 \epsilon+\frac{2}{3} \Omega\right)^{-1}\left(\frac{q+2 \epsilon}{2 \zeta+\tau_{0} \Pi}\right) .
$$

In this account, once we have made the interpretation of the causal transportation equations and then, coupled with the dynamical equation, we found the factor $1-\Lambda+\Delta$; the roll of this factor reduced the inertial energy density and effective gravitational mass density that appears in the system. This consequence agrees with the findings of Herrera et al. [35].

\section{Conclusions}

The beginning of the 20th century, after presenting general theory of relativity Weyl [58] and Levi-Civita [59] discussed the current issues with static cylindrical symmetric structures. In the starting scenario astrophysicists were focused to find the self-gravitating models that are axially symmetric. The relativistic fluids having dissipative nature are very useful in the formulation of stellar models. Therefore, one can not disagree with the effects of dissipation during the selfgravitational collapse.

In this format, we have arranged the dynamical equation which plays the meaningful role in progressive stages of the viscous dissipative gravitational collapse. We notice the impacts of $f(R)$ terms in the dynamical progression for the dissipative case; this dissipation is undergone in the form of heat flow and free streaming approximation. We have considered the convenient formation of the collapsing 
variables involved in the transportation equations in terms of heat flux, bulk, and shear viscosity ensuing from causal thermodynamical approach. Moreover, the dynamical equation is coupled with full causal transportation equations for getting the meaningful results that appear in the form of heat dissipation, bulk, and shear viscosity. In extensive point of view, the main debate of the general thermodynamical approach is to notice the relaxation phase whose direction either less than or equal to radiating time. It is interesting to mention that the hyperbolic concept of the collapse is much consistent and having rare problems than parabolic concept [60-62].

A full causal technique has been implemented in [35], to evaluate the impacts on collapsing variables with spherically symmetric collapse geometry: this study gives the expressive and momentous contribution in relativistic physics. The application of this work is obtained by some astral objects and relativistic structures. These massive systems have possessive nature in dissipative collapsing situation in which dissipation is undergone in the form of heat flow and radiation streaming. The system was weakened due to leaving the energy from the system and continues collapse. Furthermore, in this connection it is worth mentioning that thermodynamical viscous/heat coupling factors have nonterminating possibility due to dissipative gravitational collapse; this framework gives the momentous foundation in the modeling of relativistic structures. In this respect we see another previous work given by [63]; a partial technique is used to discuss the impacts of bulk dissipative gravitating collapse in cylindrical surface of the star with leaving the thermodynamical heat radiating coupling factors in the transport equations.

Finally, in this evaluation we analyze the full causal technique with viscous dissipative gravitational collapse and get the dynamical equation (74), which explain how passive gravitational mass and heat coupling factors influence the gravitational collapse of radiating source. The passive gravitational mass density is affected by bulk and shear viscosity and these factors are responsible for decreasing the internal radial pressure of the collapsing source. These factors also decrease the force of gravity in a system, this lessening due to huge dissipation in the form of thermal conduction of the gravitational collapse. Consequently, the outflow of heat reduces the total energy of the collapsing cylindrical object. Herrera et al. [64] investigated the bouncing action predicted in mathematical framework for the current arithmetic calculation. The present work is reproduced in future with other modified theories such as Gauss-Bonnet and $f(R, T)$ gravity theories. This work has been done for spherical symmetry with and without electromagnetic field [65].

\section{Appendix}

$$
\begin{aligned}
T_{01}^{D} & =\frac{1}{\kappa}\left(\dot{F}^{\prime}-\frac{A^{\prime} \dot{F}}{A}-\frac{\dot{B} F^{\prime}}{B}\right), \\
T_{11}^{D} & =\frac{B^{2}}{\kappa}\left\{\left(\frac{f(R)-R F(R)}{2}\right)+\frac{\ddot{F}}{A^{2}}+\frac{\dot{F}}{A^{2}}\left(\frac{\dot{C}}{C}+\frac{\dot{D}}{D}\right.\right. \\
& \left.\left.-\frac{\dot{A}}{A}\right)-\frac{F^{\prime}}{B^{2}}\left(\frac{A^{\prime}}{A}+\frac{C^{\prime}}{C}+\frac{D^{\prime}}{D}\right)\right\},
\end{aligned}
$$

$$
\begin{aligned}
& D_{1}=\frac{A}{\kappa}\left[\left\{\frac{1}{A^{2} B^{2}}\left(\dot{F}^{\prime}-\frac{A^{\prime} \dot{F}}{A}-\frac{\dot{B} F^{\prime}}{B}\right)\right\}_{, 1}\right. \\
& +\left\{\frac{f(R)-R F(R)}{2 A^{2}}-\frac{F^{\prime \prime}}{A^{2} B^{2}}\right. \\
& +\frac{\dot{F}}{A^{4}}\left(\frac{\dot{B}}{B}+\frac{\dot{\mathrm{C}}}{\mathrm{C}}+\frac{\dot{D}}{D}\right) \\
& \left.-\frac{F^{\prime}}{A^{2} B^{2}}\left(\frac{C^{\prime}}{C}+\frac{D^{\prime}}{D}-\frac{B^{\prime}}{B}\right)\right\}_{, 0} \\
& +\frac{2 \dot{A}}{A^{3}}\left\{\frac{f(R)-R F(R)}{2}-\frac{F^{\prime \prime}}{B^{2}}\right. \\
& \left.+\frac{\dot{F}}{A^{2}}\left(\frac{\dot{B}}{B}+\frac{\dot{C}}{C}+\frac{\dot{D}}{D}\right)-\frac{F^{\prime}}{B^{2}}\left(\frac{C^{\prime}}{C}+\frac{D^{\prime}}{D}-\frac{B^{\prime}}{B}\right)\right\} \\
& +\frac{\dot{B}}{A^{2} B}\left\{-\frac{\ddot{F}}{A^{2}}-\frac{F^{\prime \prime}}{B^{2}}+\frac{\dot{F}}{A^{2}}\left(\frac{\dot{A}}{A}+\frac{\dot{B}}{B}\right)\right. \\
& \left.+\frac{F^{\prime}}{B^{2}}\left(\frac{A^{\prime}}{A}+\frac{B^{\prime}}{B}\right)\right\}+\frac{\dot{C}}{A^{2} C}\left\{-\frac{\ddot{F}}{A^{2}}+\frac{\dot{F}}{A^{2}} \frac{\dot{A}}{A}\right. \\
& \left.+\frac{F^{\prime}}{B^{2}} \frac{A^{\prime}}{A}\right\}+\frac{\dot{D}}{A^{2} D}\left\{-\frac{\ddot{F}}{A^{2}}+\frac{\dot{F}}{A^{2}} \frac{\dot{A}}{A}+\frac{F^{\prime}}{B^{2}} \frac{A^{\prime}}{A}\right\} \\
& +\frac{1}{A^{2} B^{2}}\left(\dot{F}^{\prime}-\frac{A^{\prime} \dot{F}}{A}-\frac{\dot{B} F^{\prime}}{B}\right)\left(\frac{3 A^{\prime}}{A}+\frac{B^{\prime}}{B}+\frac{C^{\prime}}{C}\right. \\
& \left.\left.+\frac{D^{\prime}}{D}\right)\right] \\
& D_{2}=\frac{B}{\mathcal{\kappa}}\left[-\left\{\frac{1}{A^{2} B^{2}}\left(\dot{F}^{\prime}-\frac{A^{\prime} \dot{F}}{A}-\frac{\dot{B} F^{\prime}}{B}\right)\right\}_{, 0}\right. \\
& +\left\{\frac{f(R)-R F(R)}{2 B^{2}}+\frac{\ddot{F}}{A^{2} B^{2}}\right. \\
& +\frac{\dot{F}}{A^{2} B^{2}}\left(\frac{\dot{C}}{C}+\frac{\dot{D}}{D}-\frac{\dot{A}}{A}\right) \\
& \left.-\frac{F^{\prime}}{B^{4}}\left(\frac{A^{\prime}}{A}+\frac{C^{\prime}}{C}+\frac{D^{\prime}}{D}\right)\right\}_{, 1}+\frac{A^{\prime}}{A B^{2}}\left\{\frac{\ddot{F}}{A^{2}}+\frac{F^{\prime \prime}}{B^{2}}\right. \\
& \left.-\frac{\dot{F}}{A^{2}}\left(\frac{\dot{A}}{A}+\frac{\dot{B}}{B}\right)-\frac{F^{\prime}}{B^{2}}\left(\frac{A^{\prime}}{A}+\frac{B^{\prime}}{B}\right)\right\} \\
& +\frac{2 B^{\prime}}{B^{3}}\left\{\frac{f(R)-R F(R)}{2}+\frac{\ddot{F}}{A^{2}}\right. \\
& \left.+\frac{\dot{F}}{A^{2}}\left(\frac{\dot{C}}{C}+\frac{\dot{D}}{D}-\frac{\dot{A}}{A}\right)-\frac{F^{\prime}}{B^{2}}\left(\frac{A^{\prime}}{A}+\frac{C^{\prime}}{C}+\frac{D^{\prime}}{D}\right)\right\} \\
& +\frac{C^{\prime}}{B^{2} C}\left\{\frac{F^{\prime \prime}}{B^{2}}-\frac{\dot{F}}{A^{2}} \frac{\dot{B}}{B}-\frac{F^{\prime}}{B^{2}} \frac{B^{\prime}}{B}\right\}+\frac{D^{\prime}}{B^{2} D}\left\{\frac{F^{\prime \prime}}{B^{2}}\right. \\
& \left.-\frac{\dot{F}}{A^{2}} \frac{\dot{B}}{B}-\frac{F^{\prime}}{B^{2}} \frac{B^{\prime}}{B}\right\}-\frac{1}{A^{2} B^{2}}\left(\frac{\dot{A}}{A}+\frac{3 \dot{B}}{B}+\frac{\dot{C}}{C}+\frac{\dot{D}}{D}\right) \\
& \left.\cdot\left(\dot{F}^{\prime}-\frac{A^{\prime} \dot{F}}{A}-\frac{\dot{B} F^{\prime}}{B}\right)\right] \text {. }
\end{aligned}
$$




\section{Data Availability}

The data used to support the findings of this study are available from the corresponding author upon request.

\section{Conflicts of Interest}

The authors declare that they have no conflicts of interest.

\section{Acknowledgments}

One of the authors, G. Abbas, appreciates the financial support from HEC, Islamabad, Pakistan, under NRPU project with Grant no. 20-4059/NRPU/R \& D/HEC/14/1217.

\section{References}

[1] J. R. Oppenheimer and H. Snyder, "On continued gravitational contraction," Physical Review A: Atomic, Molecular and Optical Physics, vol. 56, no. 5, pp. 455-459, 1939.

[2] C. W. Misner and D. H. Sharp, "Relativistic equations for adiabatic, spherically symmetric gravitational collapse," Physical Review A: Atomic, Molecular and Optical Physics, vol. 136, no. 2, pp. B571-B576, 1964.

[3] P. C. Vaidya, "The gravitational field of a radiating star," Proceedings of the Indian Academy of Science, vol. A33, p. 264, 1951.

[4] S. Capozziello, "Curvature quintessence," International Journal of Modern Physics D, vol. 11, pp. 483-492, 2002.

[5] M. Sharif and Z. Yousaf, "Stability of a Class of Non-Static Axial Self-Gravitating Systems in $f(R)$ Gravity," Astrophysics and Space Science, vol. 352, p. 943, 2014.

[6] M. K. Mak, T. Harko, and R. Soc, "Anisotropic Stars in General Relativity," Proceedings of the Royal Society, vol. A459, p. 393, 2003.

[7] L. Herrera and N. O. Santos, "Jeans mass for anisotropic matter," The Astrophysical Journal, vol. 438, p. 308, 1995.

[8] F. Weber, Pulsars as Astrophysical Laboratories for Nuclear and Particle Physics, vol. BS1, Bristol, UK, Institute of Physics Publishing, Dirac House, Temple Back, 1999.

[9] S. Chakraborty, S. Chakraborty, and U. Debnath, "Role of Pressure in Quasi-Spherical Gravitational Collapse," International Journal of Modern Physics, vol. D14, p. 1707, 2005.

[10] R. Garattini, "Naked Singularity in a Modified Gravity Theory," Journal of Physics: Conference Series, Article ID 012066, 2009.

[11] M. Sharif and M. Zaeem Ul Haq Bhatti, "Structure scalars for charged cylindrically symmetric relativistic fluids," General Relativity and Gravitation, vol. 44, no. 11, pp. 2811-2823, 2012.

[12] M. Sharif and M. Z. Bhatti, "Structure scalars in charged plane symmetry," Modern Physics Letters A, vol. 27, no. 27, p. 1250141, 2012.

[13] M. Sharif and Z. Yousaf, "Evolution of expansion-free selfgravitating fluids and plane symmetry," International Journal of Modern Physics, vol. D21, Article ID 1250095, 2012.

[14] M. Sharif and Z. Yousaf, "Expansion-free Cylindrically Symmetric Models," Canadian Journal of Physics, vol. 90, p. 865, 2012.

[15] M. Sharif and H. R. Kausar, "Effects of $f(R)$ model on the dynamical instability of expansionfree gravitational collapse," Journal of Cosmology and Astroparticle Physics, vol. 7, p. 022, 2011.
[16] M. Sharif and M. Azam, "Effects of Electromagnetic Field on the Dynamical Instability of Cylindrical Collapse," Journal of Cosmology and Astroparticle Physics, vol. 02, p. 043, 2012.

[17] M. Sharif and S. Fatima, "Charged cylindrical collapse of anisotropic fluid," General Relativity and Gravitation, vol. 43, no. 1, pp. 127-142, 2011.

[18] M. Sharif and G. Abbas, "Dynamics of Non-adiabatic Charged Cylindrical Gravitational Collapse," Astrophysics and Space Science, vol. 335, p. 515, 2011.

[19] G. Cognola, E. Elizalde, S. Nojiri, S. D. Odintsov, and S. Zerbini, "One-loop $\mathrm{f}(\mathrm{R})$ gravity in de Sitter universe," Journal of Cosmology and Astroparticle Physics, vol. 02, Article ID 010, p. 010, 2005.

[20] S. Capozziello and M. de Laurentis, "Extended Theories of Gravity," Physics Reports, vol. 509, no. 4-5, pp. 167-321, 2011.

[21] E. J. Copeland, M. Sami, and S. Tsujikawa, "Dynamics of dark energy," International Journal of Modern Physics D, vol. 15, no. 11, pp. 1753-1936, 2006.

[22] L. Amendola, R. Gannouji, D. Polarski, and S. Tsujikawa, "Conditions for the cosmological viability of $f(R)$ dark energy models," Physical Review D: Particles, Fields, Gravitation and Cosmology, vol. 75, no. 8, Article ID 083504, 2007.

[23] S. Chandrasekhar, "The dynamical instability of gaseous masses approaching the Schwarzschild limit in general relativity," The Astrophysical Journal, vol. 140, pp. 417-433, 1964.

[24] L. Herrera, G. le Denmat, and N. O. Santos, "Dynamical instability for nonadiabatic spherical collapse," Royal Astronomical Society. Monthly Notices, vol. 237, no. 2, pp. 257-268, 1989.

[25] G. Abbas, S. Nazeer, and M. A. Meraj, "Cylindrically symmetric models of anisotropic compact stars," Astrophysics and Space Science, vol. 354, p. 449, 2014.

[26] M. K. Mak and T. Harko, "Quark stars admitting a oneparameter group of conformal motions," International Journal of Modern Physics, vol. D13, p. 149, 2004.

[27] F. Rahaman, R. Sharma, S. Ray, R. Maulick, and I. Karar, "Strange stars in Krori-Barua space-time," The European Physical Journal C, vol. 72, p. 2071, 2012.

[28] L. Herrera, "Cracking of self-gravitating compact objects," Physics Letters A, vol. 165, no. 3, pp. 206-210, 1992.

[29] L. Herrera, D. Prisco, and A. Ibanez, "On the Role of Electric Charge and Cosmological Constant in Structure Scalars," Physical Review A, vol. D84, p. 107501, 2011.

[30] L. Herrera, J. Ospino, and A. D. Prisco, "All static spherically symmetric anisotropic solutions of Einstein's equations," Physical Review, vol. D77, p. 027502, 2008.

[31] L. Herrera, N. O. Santos, and A. Wang, "Shearing Expansionfree Spherical Anisotropic Fluid Evolution," Physical Review, p. D78, 2008.

[32] L. Herrera and N. O. Santos, "Dynamics of dissipative gravitational collapse," Physical Review, vol. D70, p. 084004, 2004.

[33] L. Herrera, G. Le Denmat, and N. O. Santos, "Dynamical instability and the expansion-free condition," General Relativity and Gravitation, vol. 44, no. 5, pp. 1143-1162, 2012.

[34] A. D. Prisco, L. Herrera, J. Ospino, N. O. Santos, and V. M. Vina-Cervantes, "Expansion-Free Cavity Evolution: Some exact Analytical Models," International Journal of Modern Physics, vol. D20, p. D20, 2011.

[35] L. Herrera, A. D. Prisco, E. Fuenmayor, and O. Troconis, "Dynamics of viscous dissipative gravitational collapse: A full causal approach," International Journal of Modern Physics, vol. D18, p. 129, 2009. 
[36] A. Di Prisco, L. Herrera, G. Le Denmat, M. A. H. MacCallum, and N. O. Santos, "Nonadiabatic charged spherical gravitational collapse," Physical Review D: Particles, Fields, Gravitation and Cosmology, vol. 76, Article ID 064017, 2007.

[37] L. Herrera, M. A. H. Maccallum, and N. O. Santos, "On the Matching Conditions for the Collapsing Cylinder," Classical and Quantum Gravity, vol. 24, p. 1033, 2007.

[38] A. Di Prisco, L. Herrera, M. A. MacCallum, and N. O. Santos, "Shearfree cylindrical gravitational collapse," Physical Review D: Particles, Fields, Gravitation and Cosmology, vol. 80, no. 6, Article ID 064031, 10 pages, 2009.

[39] B. C. Nolan, "Naked singularities in cylindrical collapse of counterrotating dust shells," Physical Review, vol. D65, p. 104006, 2002.

[40] S. A. Hayward, "Gravitational waves, black holes and cosmic strings in cylindrical symmetry," Classical and Quantum Gravity, vol. 17, p. 1749, 2000.

[41] I. Müller, "Zum Paradoxon der Wärmeleitungstheorie," Zeitschrift für Physik, vol. 198, no. 4, pp. 329-344, 1967.

[42] W. Israel, "Nonstationary irreversible thermodynamics: a causal relativistic theory," Annals of Physics, vol. 100, no. 1-2, pp. 310331,1976

[43] W. Israel and J. M. Stewart, "Transient relativistic thermodynamics and kinetic theory," Annals of Physics, vol. 118, no. 2, pp. 341-372, 1979.

[44] R. Maartens, "Causal Thermodynamics in Relativity" Astrophysics, Article ID 9609119.

[45] R. Chan, "Radiating gravitational collapse with shear viscosity," Monthly Notices of the Royal Astronomical Society, vol. 316, p. $588,2000$.

[46] K. S. Thorne, "Energy of infinitely long, cylindrically symmetric systems in general relativity," Physical Review B: Condensed Matter and Materials Physics, vol. 138, p. B251, 1965.

[47] S. M. Shah and G. Abbas, "Dynamics of charged viscous dissipative cylindrical collapse with full causal approach," European Physical Journal, vol. A53, p. 53, 2017.

[48] T. Chiba, "Cylindrical dust collapse in general relativity: toward higher dimensional collapse," Progress of Theoretical and Experimental Physics, vol. 95, no. 2, pp. 321-338, 1996.

[49] H. Chao-Guang, "Charged static cylindrical black hole," Acta Physica Sinica, vol. 4, no. 8, pp. 617-630, 1995.

[50] G. Darmois, Memorial des Sciences Mathematiques, vol. 25, Gautheir-Viuars, Paris, France, 1927.

[51] S. Guha and R. Banerji, "Dissipative cylindrical collapse of charged anisotropic fluid," International Journal of Theoretical Physics, vol. 53, no. 7, pp. 2332-2348, 2014.

[52] J. M. M. Senovilla, "Junction conditions for $F(R)$-gravity and their consequences," Physical Review, vol. 88, p. 064015, 2013.

[53] T. Clifton, P. K. S. Dunsby, R. Goswami, and A. M. Nzioki, "On the absence of the usual weak-field limit, and the impossibility of embedding some known solutions for isolated masses in cosmologies with $f(R)$ dark energy," Physical Review, vol. D87, p. 063517, 2013.

[54] N. Deruelle, M. Sasaki, and Y. Sendouda, "Junction Conditions in $f(R)$ Theories of Gravity," Progress of Theoretical and Experimental Physics, vol. 119, p. 237, 2008.

[55] A. Ganguly, R. Gannouji, R. Goswami, and S. Ray, "Neutron stars in the Starobinsky model," Physical Review D: Particles, Fields, Gravitation and Cosmology, vol. 89, no. 6, 2014.
[56] R. Goswami, A. M. Nzioki, S. D. Maharaj, and S. G. Ghosh "Collapsing spherical stars in $f(R)$ gravity," Physical Review D, vol. 90, no. 8, 2014.

[57] C. W. Misner and D. Sharp, "Relativistic equations for spherical gravitational collapse with escaping neutrinos," Physical Review, vol. B137, p. 1360, 1965.

[58] H. Weyl, "On the Theory of Gravitation," Annalen der Physik (Leipzig), vol. 54, p. 117, 1917.

[59] T. Levi-Civita, "d2 einsteiniani in campi newtoniani. IX. L'analogo del potenziale logaritmico," Atti Della Reale Accademia Dei Lincei. Rendiconti, vol. 28, p. 101, 1919.

[60] C. Cattaneo, "Sulla conduzione del calore," Atti del Seminario Matematico e Fisico dell' Universita di Modena e Reggio Emilia, vol. 3, p. 3, 1948.

[61] A. Anile, D. Pavon, and V. Romano, "The Case for Hyperbolic Theories of Dissipation in Relativistic Fluids," https://arxiv.org/ abs/gr-qc/9810014.

[62] L. Herrera and D. Pavon, "Hyperbolic theories of dissipation: Why and when do we need them?" Physica, vol. A307, p. 121, 2002.

[63] S. M. Shah and G. Abbas, "Dynamics of charged bulk viscous collapsing cylindrical source with heat flux," Journal of Physics, vol. C77, p. 251, 2017.

[64] L. Herrera, A. Di Prisco, and W. Barreto, "Thermoinertial bouncing of a relativistic collapsing sphere: A numerical model," Physical Review D: Particles, Fields, Gravitation and Cosmology, vol. 73, no. 2, 2006.

[65] G. Abbas and H. Nazar, Dissipative and Viscous Gravitational Collapse in $\mathrm{f}(\mathrm{R})$ Gravity With Full Causal Approach (submitted for publication 2018). 

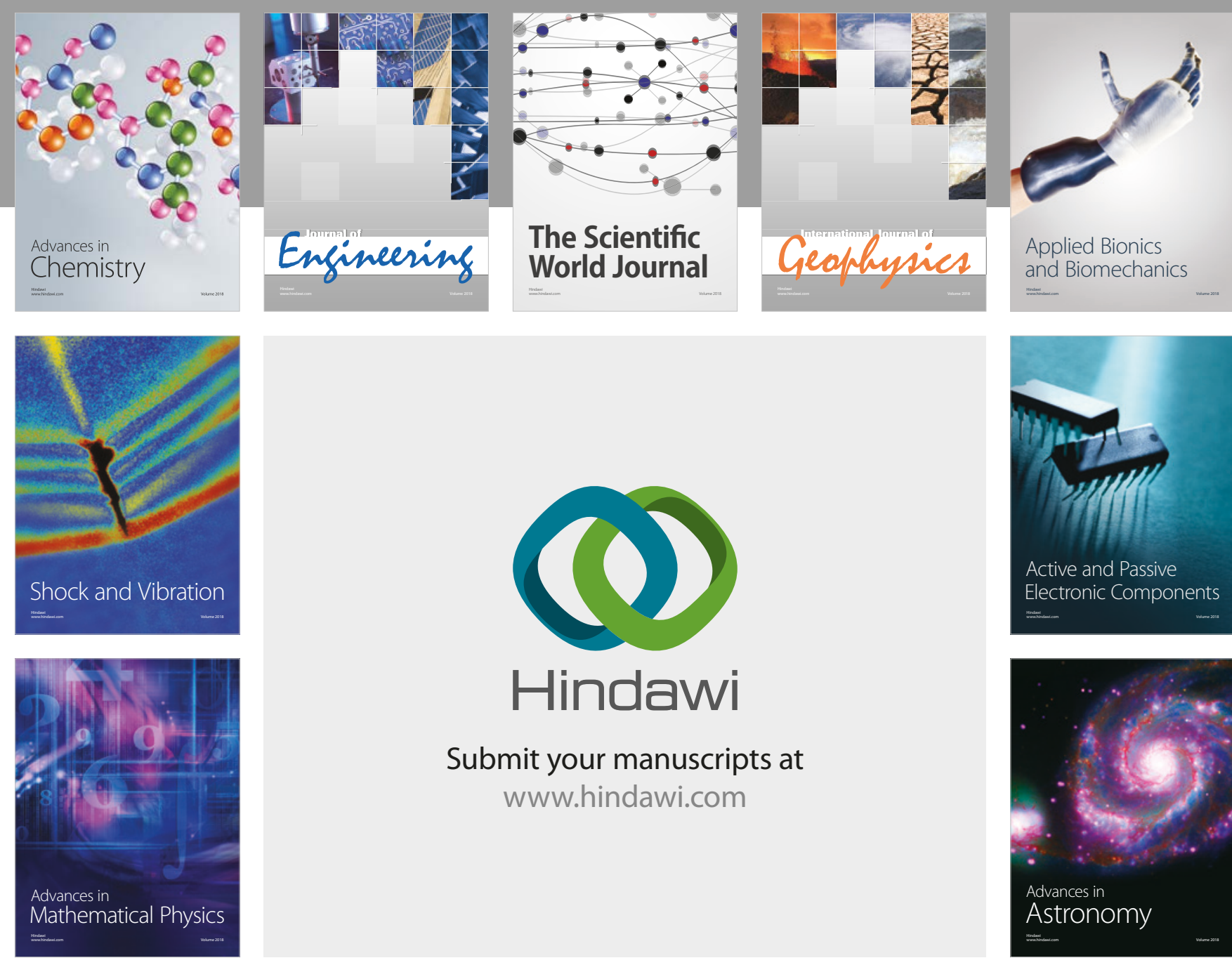

Submit your manuscripts at

www.hindawi.com

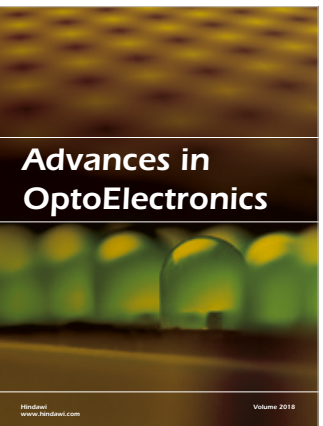

\section{Rotcting Machinery}
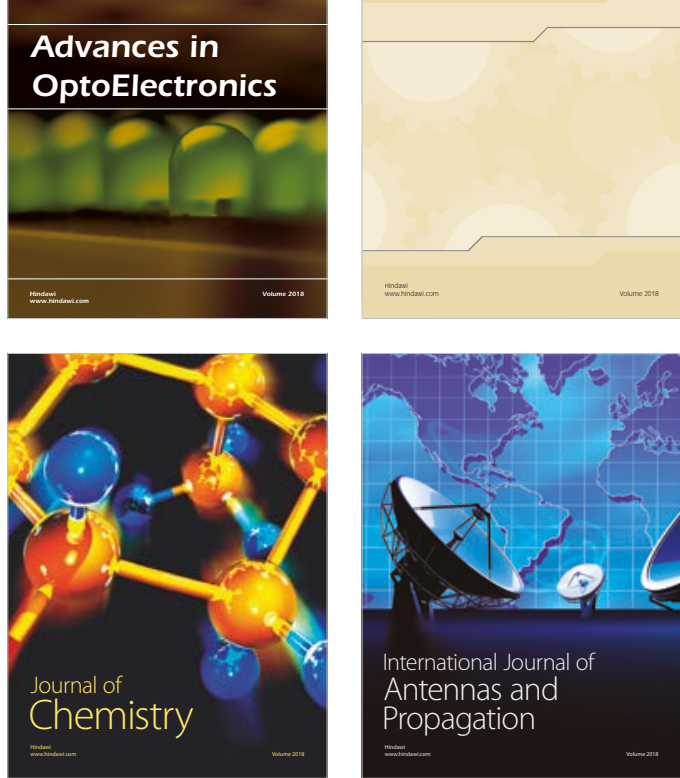

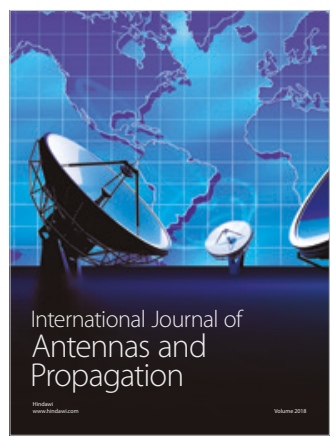

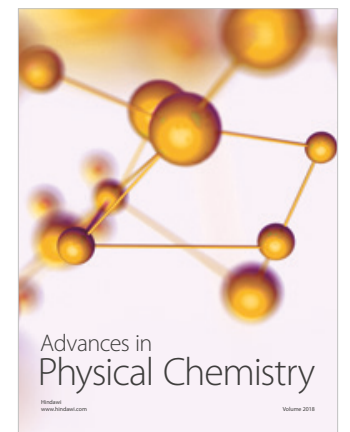

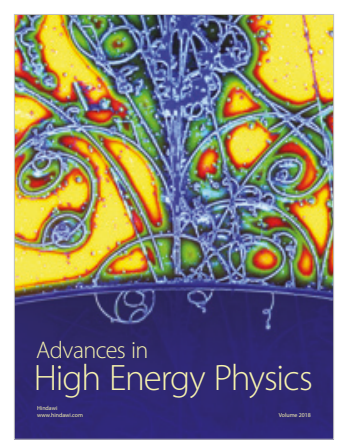

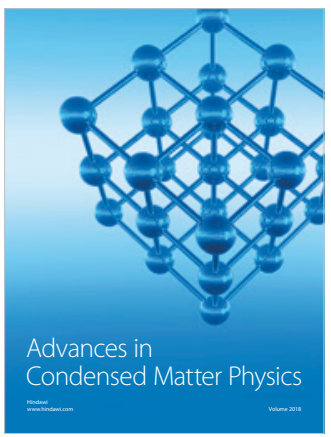

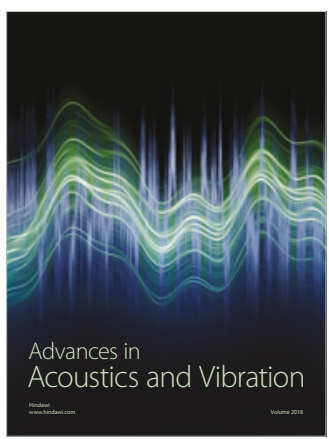

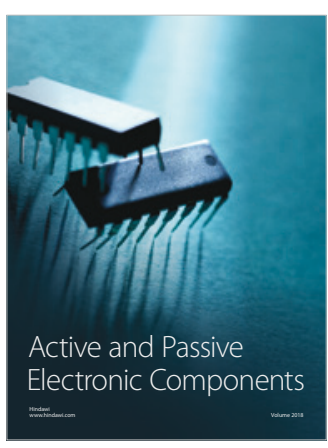
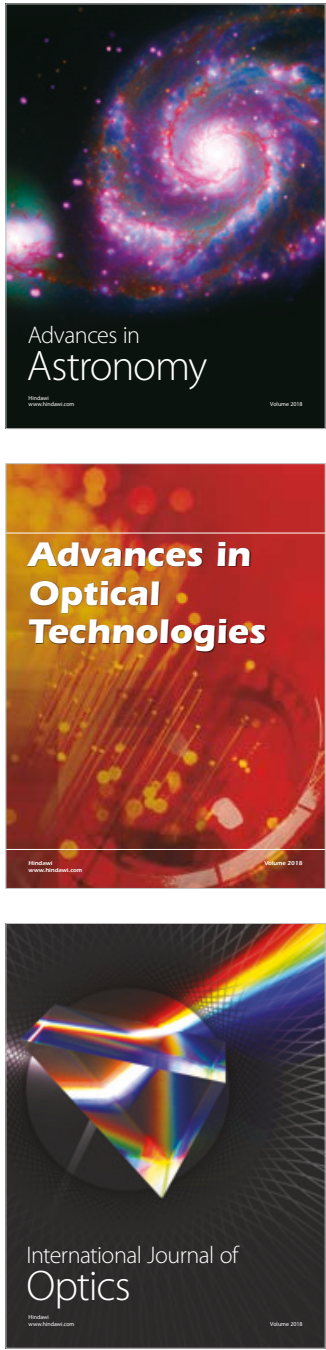